\title{
A STUDY ON MATHEMATICAL AND STATISTICAL SIMULATION APPLIED TO COVID19
}

\author{
Jaydip Datta
}

\begin{abstract}
-
In this combinatorial study let us try to simulate the four cases starting from viral spreading kinetics, Gaussian distribution of the infectious disease, modelling of remdesivir on the basis of molecular bonding approach and finally the most important mortality statistics like infection fatality ratio ( IFR ) with the distribution of age of the patient through sigmoid regression method .
\end{abstract}

Keywords - Covid19 ,SARS-COV-2, Simulation, Exponential, Gaussiun, Drug intermediate, Drug modelling, Quantum Chemistry, LCAO, Infection fatalality rate, Logistic regression, Sigmoid model, Hydrodynamic modelling, Bio-polymer, First order kinetics .

\section{Literature review -}

In my earlier work by Datta $\boldsymbol{J}$ I have reviewed the three basic mathematical modelling related to Covid19 pointing out exponential series, Gaussiun distribution function of one dimension, Probability and Probability density function, Linear combination of atomic orbital starting from wave function and area under the curve of gaussiun as well as flat gaussiun or Pseudo-gaussiun methods .The fourth work is in continuation of infection fatality rate ( IFR ) vs Age of Mallapaty $\boldsymbol{S}$ (Data Source ) in different statistical angle of best fit analysis indication logistic or Sigmoid probability .

\section{INTRODUCTION -}

To start four cases will be discussed as per following order -

Case I - Viral spreading kinetic study :

Most of the microbes including virus should follow four stages namely - lag phase, LOG or exponential phase, stationary phase and death phase . Under this present case of Covid19 the exponential phase is more prominent for spreading of infection as well as sharp one .This exponential phase may be shifted to a flat one by a prolonged lock down may decrease the infection susceptibility. This type of Covid infection then enters to a prolonged static phase of 14days or more depending on mild to severe infection for symptomatic or asymptomatic patients. The declining or death phase will be mathematically an asymptotes ie infinite time will be required to irradiate to susceptibility of infection .

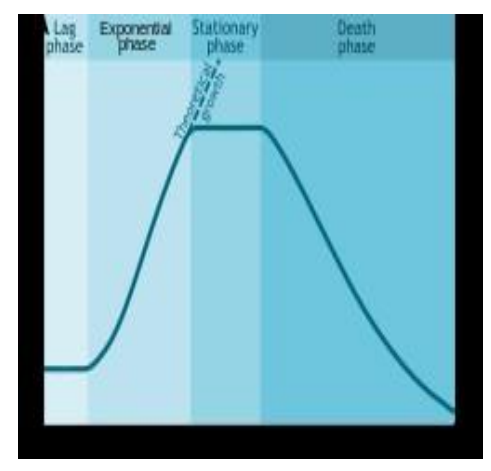


Case II - Viral spreading distribution study :

SARS-COV-2 being a virulent viral biopolymer ( like other viruses ) the distribution probability is a Gaussian one with conventional exponential phase generates a expo-gaussiun contour or pseudo Gaussiun contour ie why the the infective speed is uncontrollable, epidemic rather pandemic .

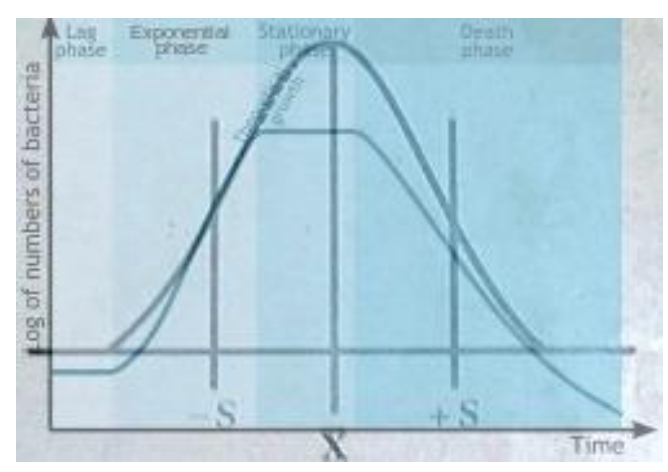

The Case I \& Case II may be included to susceptibility study of SARS-COV-2 infection .

Case III - Mortality statistics in terms of infection fatality rate ( IFR ) with age of the patients. The corresponding best fit analysis shows a logistic or sigmoid regression model. The sharp S rise of the model supports the conventional exponential phase .
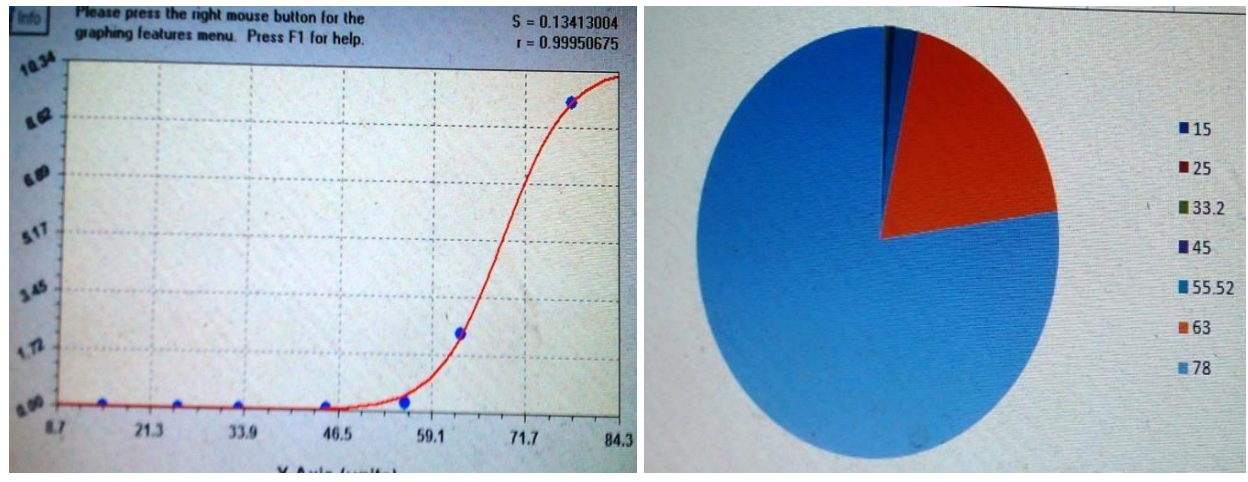

The best fit analysis plot as well as excel pie chart shows the infectivity or fatality of infection probability vs Age. Obviously the inflection data changes from 46 yrs to an old age patient of more than 70 yrs are having much more prone to death .

The Case III may be considered as infectivity study of SARS-COV-2 .

\section{Cease IV :}

It is related to novel drug modelling hypothesis of the most effective use of Covid19 antiviral like Remdesivir. In this hypothesis we will analyse the Linear combination of atomic orbital ( LCAO ) to overlap s -Orbital and p-Orbital to give rise to a hybrid Sp orbital having $50 \% \mathrm{~s}$ and $50 \% \mathrm{p}-$ Character. Like s -Orbital - spherically symmetrical like Covid19 geometry \& Remedesivir the most potent Synthetic -antiviral may be assumed to dumble shaped p-Orbital in geometry. Here Covid Remedesivir drug - Protein [DP ] intermediate may be considered Sp hybridisation analogous to 
Molecular Orbital Theory . The following logic of Atomic theory can be correlated to Covid19 as follows :

\section{MOLECULAR SIMULATION :}

A Combined Model for Covid19 (s-Orbital ) + Remedesivir ( p-Orbital ) ----------- $\rightarrow$

Covid -Remedesivir ( A SP Hybridised Model Hypothesis ) .

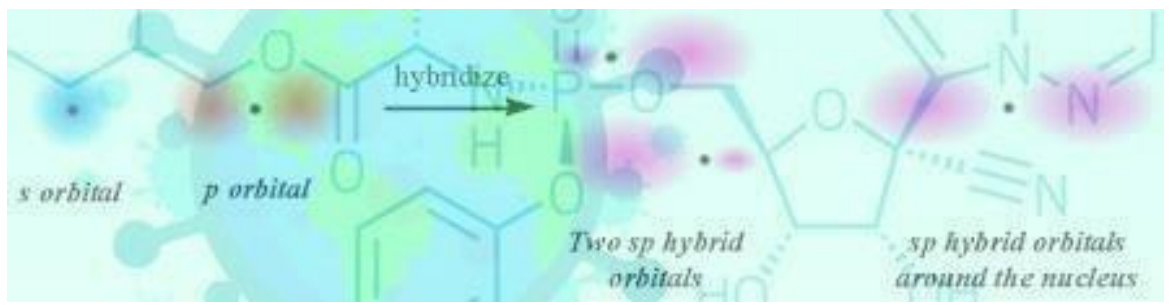

Case IV may be simulated to recovery modelling as a potent antiviral of SARS-COV-2 as mentioned above .

\section{METHODOLOGY -}

Basically includes model equations \& hypothesis in each case.

The exponential viral growh kinetics shows a Ist order kinetics as mentioned $\mathrm{Ct}=\mathrm{C} 0$ * EXP $(-\mathrm{K} * \mathrm{t})$ - -

[ Where $\mathrm{C} 0=$ Initial bacterial count, bacterial Colony Count $(\mathrm{Ct})$ at time $\mathrm{t} \& \mathrm{~K}=\mathrm{Ist}$ order growth const $\& \mathrm{t}=$ time of growth ]

General mathematical expression of Gaussiun Probability distribution of biopolymer can be expressed as :

$P(x)=A \exp \left(-B^{*} x^{\wedge} 2\right)$

A,B are constants depending on the biochemical nature of polymer chain .

Cosidering one dimension gaussiun probability $\mathrm{P}(\mathrm{x})$ ranging from - infinity to + infinity with mean distribution is zero .For two /three dimension the expression includes dimension factor . To find best fit analysis through Regression method the Gaussian fit is one of the useful one for hydrodynamic modelling of Covid19 ie the flow of virus inside Blood .

The Gaussiun probality can be represented as randomisation of monomer chain of biopolymers ( Including Virus like Covid19) ( $\mathrm{N}=$ Number of Monomers vs Rrms = An Important Statistical parameters like Root mean square end to end separation as a F(X) of Radius of gyration ( $\mathrm{Rg})$. The representative equation is as follows .

$\mathrm{P}(\mathrm{N}, \mathrm{x})=\mathrm{A} * \exp \left(-0.042 * \mathrm{Rrms}^{\wedge} 2\right) \ldots \ldots \ldots \ldots .(3)$ [ Case $-\mathrm{II}$ ]

$\mathrm{X}=$ distribution chain length of random biopolymer coil

From Cuver finder V.1.4 the best fit equation :

$\mathrm{X}($ Age $)=$ Sigmoid $\mathrm{f}[\mathrm{Y}(\mathrm{IFR})]=\left[\mathrm{a} /\left(1+\mathrm{b}^{*} \exp (-\mathrm{cx})\right]------(4)[\right.$ Case $-\mathrm{III}]$ $\mathrm{a}, \mathrm{b}, \mathrm{c}$ are the coefficients obtained from best fit analysis .

The equation ( 4 ) represents the desired model equation and the measure of a sigmoid probability of Covid infection with a significant correlation ( $\mathrm{r}$ ) value $[0<\mathrm{r}<1$ ] 
Hypothesis regarding Remdesivir antiviral drug-neucleocapsid protein of Covid19 -Quantum molecular bonding approach of drug-design . ( Case -IV )

The Theorem of Normalisation - Integration of Shi * Shi $d V=$ Unity . Very useful in Quantum Chemistry $. \mathrm{dv}=$ volume element for a probability Shi*Shi .Considering Covid19 as an uniform sphere we can compare with the spherical symmetrical s - Orbital in Atomic structure. Wave particle duality hypothesis assumes a wave nature for quick viral spreading as well as crown like particle. We can apply the probability density theory on $\mathrm{s}$ - Orbital where $\mathrm{dv}=4 / 3 *$ pie $^{*} \mathrm{R}^{\wedge} 3$.

Applying Cylindrical polar symmetry on spike crown ( R, Theta , Phi ) - The cylindrical probability can be calculated as usual.

Now considering Remedesivir as p-orbital, we can assume the [Drug- Protein] Complex as Sp

hybridisation for linear overlap .

The modelling relationship from LCAO ( Linear combination of Atomic Orbital ) -

Shi $($ Covid19 ) + Shi ( Remedesivir ) = Shi ( Drug- Protein Intermediate ). [Where Shi represents the Corresponding wave functional probability ] correlating the stability of [ R-P] complex intermediate through the approach of molecular bonding .

\section{CONCLUSION -}

Between four cases I - II may be simulated as susceptibility, case-III may be simulated as infectivity of Covid19 and IV may be considered as probability of Remdesivir- Covid19 complex resulting recovery approach of the patient

\section{REFERENCE :}

1. INDUSTRIAL MICROBIOLOGY by PRESCOTT \& DUNN .

2. Image Source - en.wkipedia.org ( microbial growth kinetics curve ), industry.com ( Covid19 ), can.acs.org ( Remdesivir ).

3. Datta J , MICROBIAL GROWTH KINETICS : A MATHEMATICAL REVIEW ( SPECIAL ADDENDUM - COVID19) ( figshare.com ), May , 2020.

4. Datta J. Gaussiun Probability : Dimensional View, ( figshare.com ), May 2020 .

\section{Datta J , HYDRODYNAMICS: AN EXPERIMENTAL VIEW ON BIO MOLECULE}

(REVISED ), osf.io, April , 2020 .

6. Datta J , https://www.researchgate.net/search (What are the BASIC mathematical methods specially used in computational chemistry covering Covid19? .

7 . . https://en.wikipedia.org/wiki/Case_fatality_rate

8. . Mallapaty Smriti - https://www.nature.com/articles/d41586-020-02483-2

$$
-4-
$$


9.https://en.wikipedia.org/wiki/Logistic_regression\#: :text=Logistic\%20regression\%20is\%20a\%20st atistical,a\%20form\%20of\%20binary\%20regression).

10 . Datta J , Preprint at https://doi.org/10.6084/m9.figshare.12907619

11. https://figshare.com/articles/dataset/Data -

_INFECTION_FATALITY_RATE_OF_COVID19_A_LOGISTIC_MODEL/12979856

12. Datta J , https://doi.org/10.31219/osf.io/wuqa6

(https://figshare.com/articles/figure/BEST_FIT_STATISTICAL_ANALYSIS_OF_BIO_POLYMERS AN_EXPONENTIAL_FIT/12264491)

13. Datta J , https://doi.org/10.6084/m9.figshare.12264491

14 .Datta J,

https://www.researchgate.net/post/What_are_the_BASIC_mathematical_methods_specially_used_in COMPUTATIONAL_CHEMISTRY_covering_Covid19

15. Datta J, COVID 19 - A COMPRENSIVE MATHEMATICAL APPROACH , ( https://doi.org/10.31219/osf.io/vgf3m )

16.

https://figshare.com/articles/MICROBIAL_GROWTH_KINETICS_A_MATHEMATICAL_REVIE W_SPECIAL_ADDENDUM_-_COVID19_/12280790, JAYDIP DATTA , May ,11, 2020 .

17. https://osf.io/c6nmt/ , JAYDIP DATTA , May ,18,2020 .

18 .https://figshare.com/articles/COVID19-chemical_bonding_pdf/12533348, JAYDIP DATTA .

19.Jaydip Datta , A STUDY ON SOME STRUCTURAL FEATURES RESPONSIBLE FOR SARS-

COV-2 INFECTION FATALITY ,January 2021,DOI: 10.31219/osf.io/m62ba .License : CC BY-ND 4.0.

(https://www.researchgate.net/publication/335564477_A_STUDY_ON_SOME_STRUCTURAL_F EATURES RESPONSIBLE FOR SARS-COV-

2 INFECTION FATALITY? sg\%5B0\%5D=7IbBJ6wwDCXT 4SJqVK30i5cmZkkjNucKAGfnt5 JdU63RC_pOknmwEZ7tP6XO5pbDf0N2HiFn4q7yvTB19CfmmMJh8R5gqlbThTZLR9d.U6kkahU oF3cYZfkkW-kqwnNhhUd2JX4dZ8C5Q03s5z9vchDDiyPBkE21syULc-cEWDujEyzlvTfcTk5pVbX_Q).

DATTA, J. (2021, January 23). A STUDY ON SOME STRUCTURAL FEATURES RESPONSIBLE FOR SARS-COV-2 INFECTION FATALITY.

https://doi.org/10.31219/osf.io/m62ba

DATTA, JAYDIP. “A STUDY ON SOME STRUCTURAL FEATURES RESPONSIBLE FOR SARS-COV-2 INFECTION FATALITY.” OSF Preprints, 23 Jan. 2021. Web.

DATTA, JAYDIP. 2021. "A STUDY ON SOME STRUCTURAL FEATURES RESPONSIBLE FOR SARS-COV-2 INFECTION FATALITY.” OSF Preprints. January 23.

doi:10.31219/osf.io/m62ba. 